\title{
Volume reflection of ultrarelativistic particles in single crystals
}

\author{
V. A. Maisheev \\ Institute for High Energy Physics, 142281, Protvino, Russia
}

(Received 9 April 2007; published 22 August 2007)

\begin{abstract}
An analytical description of volume reflection of charged ultrarelativistic particles in bent single crystals is considered. The relation describing the angle of volume reflection as a function of the transversal energy is obtained. Different angle distributions of the scattered protons in single crystals are found. Results of calculations for $400 \mathrm{GeV}$ protons scattered by the silicon single crystal are presented.
\end{abstract}

DOI: 10.1103/PhysRevSTAB.10.084701

PACS numbers: $61.85 .+\mathrm{p}$

\section{INTRODUCTION}

Volume reflection of charged particles in single crystals represents the coherent scattering of these particles by planar or axial electric fields of bent crystallographic structures. For the first time, this effect was predicted in Refs. [1,2]. An angle of volume reflection is small enough (of the order of the critical angle of channeling). Because of this, it is thought that this process did not show itself in experimental measurements. However, in the recent reports $[3,4]$ problems were discussed, which require the existence of the volume reflection effect for explanation of the proton and heavy ion collimation measurements. Besides, the preliminary results of the experimental observation of volume reflection were published [5,6].

The recent meetings in CERN [7] which were devoted to problems of utilization of interactions of proton beams with single crystals attract considerable interest to the phenomenon of volume reflection due to new possibilities such as the collimation [8-10] and extraction [11] of collider beams, creation of devises which can multiply the effect [12] and so on.

In September 2006 at the CERN $400 \mathrm{GeV} / c$ extracted proton beam experiment, an effect of volume reflection was observed and studied for various silicon single crystals [13]. The use of the unique measurement technique provided a possibility to determine parameters of the process with very high precision. In the immediate future, these experimental works will continue on proton beams in CERN [14] and IHEP [15].

By now, basic results of the theoretical investigation of volume reflection are mainly based on Monte Carlo calculations. No doubt, this is a very powerful method. However, an analytical solution of the problem is very useful for understanding the process and its different characteristics. In this paper we consider such a description of the volume reflection effect.

It is well known that an ordered location of atoms in single crystals creates periodic microscopic electric fields. The entering charge particle in a single crystal moves under interaction with these fields. However, the motion of the particle is perturbed by multiple scattering on atoms and electrons. Because of this, the character of particle motion in single crystals becomes more complicated.

Taking into account that mentioned above, we start to investigate the unperturbed motion of charge particles in bent single crystals. It allows us to find various characteristics of the volume reflection process. After that, we consider multiple scattering and obtain an expansion of the results for this case.

The paper is organized as follows. First, we describe an analytical model of the phenomenon. Based on the equations of particle motion in bent single crystals, we find a formula for the angle of volume reflection and analyze its properties. After that, we find relations describing the angle distributions of scattered particles and then consider multiple scattering of particles in the body of a single crystal and investigate its influence on the volume reflection characteristics.

In the second part, we illustrate the obtained equations by numerical calculations.

It should be noted that, in this paper, we do not make the comparison of our consideration with data which were obtained in the recent experiment in CERN [13]. The reasons are the following: first, these data now are treated and hence, unpublished; second, we plan to carry out such a comparison in the frame of a future paper which will be devoted to a detailed description of the CERN experiment results.

\section{MOTION IN BENT SINGLE CRYSTALS}

One can describe the motion of ultrarelativistic particles in bent single crystals with the help of the following equations $[16,17]$ :

$$
\begin{gathered}
E_{0} \beta^{2} v_{r}^{2} /\left(2 c^{2}\right)+U(r)+E_{0} \beta^{2}(R-r) / r=E=\mathrm{const} \\
d y / d t=v_{y}=\mathrm{const} \\
v_{z}=r d \phi / d t \approx c\left(1-\frac{1}{2 \gamma^{2}}-\frac{\left(v_{r}^{2}+v_{y}^{2}\right)}{2 c^{2}}\right)
\end{gathered}
$$

These equations take place for the cylindrical coordinate 
system $(r, \phi, y)$. Here $v_{r}$ is the component of particle velocity in the radial direction, $v_{y}$ is the component of the velocity along the $y$ axis, $v_{z}$ is the tangential component of the velocity, $R$ is the radius of the bending of a single crystal, $E_{0}$ and $\gamma$ are the particle energy and its Lorentz factor, $E$ is the constant value of the radial energy, $U(r)$ is the one-dimensional potential of a single crystal, $c$ is the velocity of light, and $\beta$ is the ratio of the particle velocity to velocity of light. In this paper, we consider the planar case when the scattering is due to the interaction of particles with the set of the crystallographic planes located normally to the $(r, \phi)$ plane. On the practice it means that $v_{y} / c \gg \theta_{a c}$ but $v_{y} / c \ll 1$ for ultrarelativistic particles, where $\theta_{a c}$ is the critical angle of axial channeling.

One can transform Eq. (1) in the following form:

$$
E_{0} \beta^{2} v_{x}^{2} /\left(2 c^{2}\right)+U(x)+E_{0} \beta^{2} x / R=E
$$

where $x$ is the local Cartesian coordinate which connected with the cylindrical coordinate $r$ through the relation $x=$ $R-r$ and $v_{x}=v_{r}$. We also changed the $r$ value in the denominator of Eq. (1) on $R$. For a real experimental situation, it brings a negligible mistake (of the order of $x / R)$. In Eq. (4) the $E$ value has a sense of the transversal energy.

The equations considered here describe the threedimensional motion of particles in a bent single crystal in the cylindrical coordinate system. Let us introduce the Cartesian coordinate system in which the $x y$ plane is coincident with the front edge of a single crystal. Now we can calculate the $x$ component of the velocity in this system:

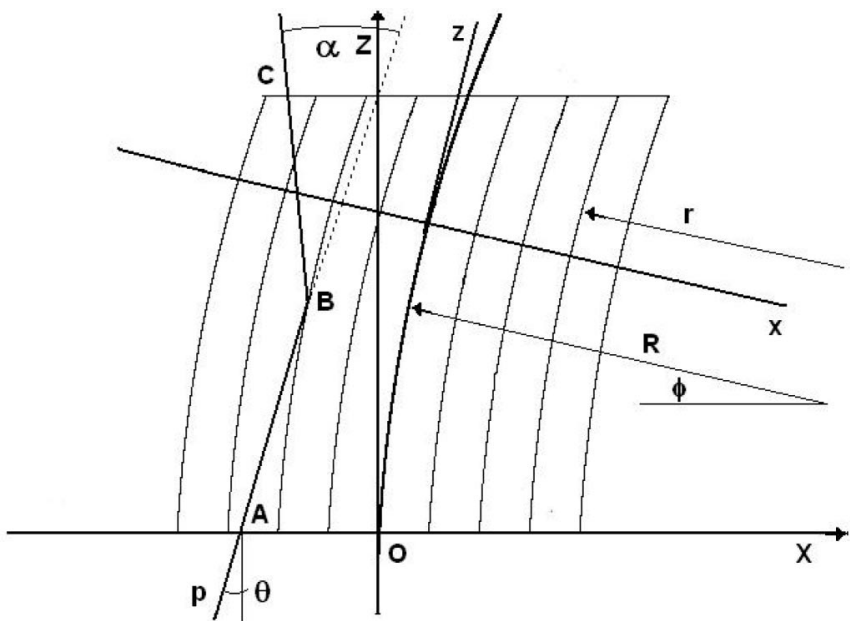

FIG. 1. Scheme of the volume reflection process. $X Y Z$ is the Cartesian coordinate system at the entrance in a single crystal, $x y z$ is the local Cartesian coordinate system connected with the current location of a particle. The $Y$-axis is directed normally to the plane of the figure. $\theta$ and $\alpha$ are the initial and volume reflection angles. $\mathrm{AB}$ and $\mathrm{BC}$ lines are the incoming and outgoing directions of a particle.

$$
V_{x}=v_{x} \cos \phi+v_{z} \sin \phi \approx v_{x}+v_{z} \phi .
$$

Figure 1 illustrates the geometry of the proton beam volume reflection and different coordinate systems which will be used in our consideration.

\section{ANGLE OF VOLUME REFLECTION}

One can see that Eq. (4) describes the one-dimensional motion in the effective potential $U_{e}(x)=U(x)+$ $E_{0} \beta^{2} x / R$. There are two different kinds of infinite motion in the effective potential. One case corresponds to motion when the particle after its entrance in the single crystal moves in the direction of the increasing of potential (in the positive direction of the $x$ axis). In this case, the velocity $v_{x}$ decreases and in some point $x_{c}$ becomes equal to zero and then the particle begins to move in the opposite direction. It corresponds to the case when the effect of volume reflection is possible. In the second case, the particle after entrance in a single crystal moves in the negative direction of the $x$ axis and the volume reflection is impossible. Obviously, a trajectory belonging to some kind of events is determined by the initial conditions of particles at the entrance in a single crystal: the first case takes place at the positive entering angles $\theta$ relative to crystallographic planes (see Fig. 1). We also will consider below the case when the absolute value of $\theta$ is larger than the channeling angle $\theta_{c}$.

One can calculate the trajectory in the $x$ direction $\left(x_{0} \leq\right.$ $x \leq x_{c}$ ) up to point $x_{c}$ as

$$
t=\int_{x_{0}}^{x} \frac{d x}{\sqrt{\frac{2 c^{2}}{E_{0} \beta^{2}}\left[E-U(x)-E_{0} \beta^{2} x / R\right]}},
$$

where $t$ is the time and $t=0$ corresponds to a coordinate $x_{0}$ at the entrance of a single crystal. We can rewrite this equation in the following equivalent form:

$$
\begin{aligned}
t= & \int_{x_{0}}^{x}\left[\frac{1}{\sqrt{\frac{2 c^{2}}{E_{0} \beta^{2}}\left[E-U(x)-E_{0} \beta^{2} x / R\right]}}-F(x)\right] d x \\
& +\int_{x_{0}}^{x} F(x) d x,
\end{aligned}
$$

where the function $F(x)$ reads

$$
F(x)=\frac{1}{\sqrt{\frac{2 c^{2}}{E_{0} \beta^{2}}\left[E-U\left(x_{c}\right)-E_{0} \beta^{2} x / R\right]}},
$$

and the critical point $x_{c}$ is determined by the equation

$$
E-U\left(x_{c}\right)-E_{0} \beta^{2} x_{c} / R=0 .
$$

Note that the equation $t=\int_{x_{0}}^{x} F(x) d x$ describes one dimension motion of a particle, which does not interact with the electric field of a single crystal. We name this case the neutral motion. 
Taking the integral $\int_{x_{0}}^{x} F(x) d x$, we get

$$
t=T\left(x_{0}, x\right)+R / c^{2}\left[\tilde{\boldsymbol{v}}\left(x_{0}\right)-\tilde{\boldsymbol{v}}(x)\right],
$$

where

$$
T\left(x_{0}, x\right)=\int_{x_{0}}^{x}\left[\frac{1}{\sqrt{\frac{2 c^{2}}{E_{0} \beta^{2}}\left[E-U(x)-E_{0} \beta^{2} x / R\right]}}-F(x)\right] d x,
$$

and $\tilde{v}(x)$ is the velocity of neutral motion in the point $x$ of its trajectory:

$$
\tilde{\boldsymbol{v}}(x)=\sqrt{2 c^{2} /\left(E_{0} \beta^{2}\right)\left[E-U\left(x_{c}\right)-E_{0} \beta^{2} x / R\right]} .
$$

The angle $\phi$ in Eq. (5) is approximately equal to $V_{z} t / R \approx V t / R \approx c t / R$. For our aims, a small difference between $V_{z}, V$, and $c$ is of no significance. We will use the relation $\phi=c t / R$. Hence, Eq. (5) has the following form:

$$
V_{x}=v_{x}+v_{z} c t / R \approx v_{x}+c^{2} t / R .
$$

Taking $t$ from Eq. (10), one can get

$$
V_{x}=v_{x}+T\left(x_{0}, x\right) c^{2} / R+\tilde{v}\left(x_{0}\right)-\tilde{v}(x) .
$$

Now we assume that the distance $d_{v r}=\left|x_{c}-x_{0}\right|$ is large enough (see estimations and calculations below). Hence, $V_{x} \approx \tilde{v}\left(x_{0}\right)\left[v_{x} \approx \tilde{v}(x), T\left(x_{0}, x\right) \approx 0\right]$ when a particle moves far from the critical point (near the $x_{0}$ point). It is easy to see that in the critical point $v_{x}=0, \tilde{v}(x)=0$, and $V_{x}=\tilde{v}\left(x_{0}\right)+T\left(x_{0}, x_{c}\right) c^{2} / R$. Using the obvious symmetrical properties of one-dimensional motion, we can get that, for a distant enough from critical point values $x>$ $x_{c} V_{x}=\tilde{v}\left(x_{0}\right)+2 T\left(x_{0}, x_{c}\right) c^{2} / R$. From here, the angle of volume reflection $\alpha=2 T\left(x_{0}, x_{c}\right) c / R$ or its explicit value has the following form:

$$
\alpha=\frac{2 c}{R} \int_{x_{0}}^{x_{c}}\left[\frac{1}{\sqrt{\frac{2 c^{2}}{E_{0} \beta^{2}}\left[E-U(x)-E_{0} \beta^{2} x / R\right]}}-F(x)\right] d x .
$$

It is easy to understand the meaning of the $F$-function introduced in Eq. (8). This function allows one to eliminate an influence of geometrical factors on particle motion in the cylindrical coordinate system. The visual picture of volume reflection may also be observed in the cylindrical coordinates $r$ and $\phi$. Really, the trajectories of the charged and neutral particles have the same critical radial coordinate $r_{c}$ but different values of $\phi_{c}$. The difference of $\phi_{c}$ coordinates determines the angle of volume reflection.

\section{PERIODICITY OF VOLUME REFLECTION ANGLE}

Considering the volume reflection angle as a function of the transversal energy $E$, one can see that this function is periodic. The period is equal to

$$
\delta E=E_{0} \beta^{2} d / R .
$$

One can get this fact by the substitution $x=x+d$ ( $d$ is the interplanar distance) and taking into account the periodicity of the potential $U(x)$.

The transversal energy $E$ has the following representation:

$$
E=E_{0} \beta^{2} \theta^{2} / 2+U\left(x_{0}\right)+E_{0} \beta^{2} x_{0} / R .
$$

The replacement $x_{0}$ on $x_{0}+d$ corresponds to new energy $E+\delta E$. It means that the angle $\alpha$ is a periodic function of the initial coordinate $x_{0}$.

The volume reflection angle is also a periodic function of the square of initial angle $\theta$. In this case the period is

$$
\delta \theta^{2}=2 d / R \text {. }
$$

It should be noted that the periodicity of the volume reflection angle relative to the above pointed parameters violated if the condition $d_{v r} \gg \theta_{c} R$ is not satisfied or, in other words, at transformation from one energy to another the distance $\left|x_{0}-x_{c}\right|$ should be large enough.

\section{PARTICLE DISTRIBUTIONS AT VOLUME REFLECTION}

In calculations, we will assume that the distribution of a particle beam over the initial coordinate $x_{0}$ is uniform (in the limits of one period). If the function $\alpha\left(x_{0}\right)\left(0 \leq x_{0} \leq\right.$ $d$ ) is known, we can find the distribution of particles over the angle of volume reflection with the help of the following relation:

$$
\frac{d N}{d \alpha}(\alpha)=\sum_{i} \frac{1}{d}\left|\frac{d \alpha}{d x_{0}}\right|^{-1},
$$

where the sum over $i$ means that the sum should be taken over every domain of the function single valuedness and the derivative $d \alpha / d x_{0}$ should be calculated for values $x_{0 i}$ which satisfy the equation $\alpha\left(x_{0 i}\right)=\hat{\alpha}$ in which $\hat{\alpha}$ is the current (computed) value of the volume reflection angle. Note Eq. (19) was obtained at the condition that the initial angle $\theta$ is fixed. Besides, in this paper we use the distribution functions which were normalized to 1 .

In actual practice, a particle beam has some distribution over the initial angles. It is obvious that the distribution function in Eq. (19) depends on the initial angle $\theta$. On the other hand, the volume reflection angle is the periodic function of the $\theta^{2}$ value. At large enough $\theta$ angles and a small range of variation of this angle, one can consider the angle $\alpha$ as a periodic function of the initial angle $\theta$ with the period:

$$
\delta \theta=\frac{d}{\theta R} .
$$

It is easy to see that $\delta \theta$ is a very small value. Simple estimations give values of $\delta \theta \sim 1-0.01 \mu \mathrm{rad}$ for radius in the range $1-100 \mathrm{~m}$, correspondingly, for the initial angle 
about $100 \mu \mathrm{rad}$ and $d$ about some angstroms. Real particle beams have a significantly more broad angle distribution. Assuming the uniform distribution of the initial angles within the angle period $\delta \theta$ (at the assumption that divergence of beam satisfied the condition $\Delta \theta \ll \theta$ ), we get the distribution averaged over its period:

$$
\langle d N / d \alpha\rangle=\frac{1}{\delta \theta} \int_{\theta}^{\theta+\delta \theta} \frac{d N}{d \alpha} d \theta .
$$

This equation is obtained for a physically narrow particle beam. Under this term, we understand the beam with the small divergence of initial angles, which in tens or more times exceed the angle period in Eq. (20). The distribution of a beam with the valuable quantity $\Delta \theta$ (however, $\Delta \theta \ll$ $\theta$ ) has the following form:

$$
\left\langle\frac{d N}{d \alpha}(\alpha)\right\rangle_{\Delta \theta}=\int_{\theta_{0}-\theta_{1}}^{\theta_{2}-\theta_{0}} \rho_{b}\left(\xi, \theta_{0}\right)\left\langle\frac{d N}{d \alpha}(\alpha-\xi)\right\rangle d \xi,
$$

where $\rho_{b}\left(\xi, \theta_{0}\right)$ is the angle distribution of the beam around the initial angle $\theta_{0}, \theta_{1}$ and $\theta_{2}$ are the minimal and maximal values of the initial angles $\left(\Delta \theta=\theta_{2}-\theta_{1}\right)$. In this equation the angle $\alpha$ is the planar angle relative to direction which is determined by $\theta_{0}$.

Now we can give another representation of the particle distribution over the volume reflection angle. For this purpose, we write the distribution function $\frac{d N}{d \alpha}$ in the following form:

$$
\frac{d N}{d \alpha}(\theta, \alpha)=\sum_{j} \frac{d N}{d E}(\theta, E)\left|\frac{d \alpha}{d E}(E)\right|^{-1},
$$

where the sum over $j$ means that the sum should be taken over every domain of the function single valuedness and the derivative $d \alpha / d E$ should be calculated for values $E_{j}$ which satisfy the equation $\alpha\left(E_{j}\right)=\hat{\alpha}$ in which $\hat{\alpha}$ is the current value of the volume reflection angle [see also Eq. (19)]. Function $\frac{d N}{d E}(\theta, E)$ is the distribution of the incoming particles (under fixed initial angle $\theta$ ) over the transversal energies. This function reads

$$
\frac{d N}{d E}(\theta, E)=\sum_{k} \frac{1}{\Delta}\left|\frac{d U_{e}\left(x_{0}\right)}{d x_{0 k}}\right|^{-1},
$$

where $U_{e}=U\left(x_{0}\right)+E_{0} \beta^{2} x_{0} / R$ and the sum should be taken over every domain of the function single valuedness and $x_{0 k}$ is the root of Eq. (17). Here $\Delta$ is the size of set $\left\{x_{0 k}\right\}$. This equation is based on the equiprobable distribution of the incoming particle over resolved coordinates and it is similar to the equation in Ref. [18] which was obtained for straight single crystals when $\Delta=d$. Note that $\Delta$ depends on the bending radius of a single crystal.

Now we can obtain the distribution function $\left\langle\frac{d N}{d \alpha}(\alpha)\right\rangle$ [see Eq. (21)]. Let us consider the transversal energies within only one period [see Eq. (20)]. Let $x_{0 k}$ be the root of Eq. (21) at the angle $\theta$ and transversal energy $E$. We can select another transversal energy $E^{\prime}$ and write the following equation:

$$
\begin{aligned}
& E_{0} \beta^{2} \theta^{2} / 2+E_{0} \beta^{2}\left(\theta_{1}^{2}-\theta^{2}\right) / 2+U_{e}\left(x_{0 k}\right) \\
& \quad=E+\left(E^{\prime}-E\right) .
\end{aligned}
$$

It is clear that, at the condition $\left(E^{\prime}-E\right)=E_{0} \beta^{2}\left(\theta_{1}^{2}-\right.$ $\left.\theta^{2}\right) / 2$, the root of this equation for a particle with the transversal energy $E^{\prime}$ and angle $\theta_{1}$ is the same as for a particle with the corresponding values $E$ and $\theta$. From here and Eq. (24), we obtain that $\frac{d N}{d E}\left(\theta, E^{\prime}\right)$ is the same [as $\frac{d N}{d E} \times$ $(\theta, E)]$ in the angle range $(\theta, \theta+\Delta \theta)$ [see Eq. (20)] but their values are shifted in between. As a result, we get that the distribution $\frac{d N}{d E}(\theta, E)$ averaged over the period of $\theta$ is equiprobable $\left[\left\langle\frac{d N}{d E}(E)\right\rangle=1 / \delta E\right]$.

Then the corresponding distribution [see Eq. (21)] has the following form:

$$
\left\langle\frac{d N}{d \alpha}(\alpha)\right\rangle=\frac{1}{\delta E} \sum_{j}\left|\frac{d \alpha}{d E}\right|^{-1}
$$

at the similar explanations as after Eq. (23). Note that the results of calculations with Eqs. (19)-(21) and (26) are equivalent.

Let us find the mean value of the angle reflection:

$$
\left\langle\alpha_{v r}\right\rangle=\int_{\alpha_{-}}^{\alpha_{+}} \alpha\left\langle\frac{d N}{d \alpha}\right\rangle d \alpha=\frac{1}{\delta E} \int_{E}^{E+\delta E} \alpha(E) d E,
$$

where $\alpha_{-}$and $\alpha_{+}$are the minimal and maximal values of $\alpha$. Here we used the previously obtained result: $\left\langle\frac{d N}{d E}(E)\right\rangle=$ $1 / \delta E$. Thus, for finding a mean angle of volume reflection (and other higher moments of the distribution), there is no necessity to know the distribution function $\langle d N / d \alpha\rangle$.

\section{INFLUENCE OF MULTIPLE SCATTERING}

The above-mentioned results of our consideration are appropriate for the ideal case only when dissipative processes in the body of a single crystal are absent. Monte Carlo calculations can give a detailed description of these processes. Here we make an attempt to take into account the influence of multiple scattering on the effect of volume reflection in the frame of analytical examination. However, in this case some realistic assumptions are needed. As was said earlier, the main contribution in the integral in Eq. (15) gives a relatively not large space area near the critical point $x_{c}$. Hence, we can neglect by multiple scattering in this area.

The process of multiple scattering on the particle path from $x_{0}$ until $\sim x_{c}$ brings the variation of the transversal energy $E$. Bearing in mind that the scattering can play the important role in a small area near the $x_{c}$ coordinate, we suppose that this factor may be taken into account by the corresponding changing of the initial data [see Eq. (17)]. We assume that the physically narrow beam has the initial angle $\theta$. Then we can consider that the initial distribution of particles is the following: 


$$
\left\langle\frac{d N}{d \alpha}(\alpha)\right\rangle_{m s}=\int_{-\infty}^{\infty} \rho(\varphi, \sigma)\left\langle\frac{d N}{d \alpha}(\alpha-\varphi)\right\rangle d \varphi
$$

where $\rho(\varphi)=\frac{1}{\sqrt{2 \pi} \sigma} \exp -\left(\varphi^{2} / 2 \sigma^{2}\right)$ is the angle distribution of multiple scattering with the parameter $\sigma$ depending on the distance $\approx\left|x_{c}-x_{0}\right| / \theta$. We also expect that the redistribution of the initial coordinates is small and do not consider this factor. Here it is important that the distribution of particles within the interplanar distance remains uniform.

Now we can obtain the angle distribution after a single crystal for the physically narrow beam:

$$
\begin{aligned}
\left\langle\frac{d N}{d \alpha}(\alpha)\right\rangle_{\mathrm{exit}} & =\int_{-\infty}^{\infty} \rho\left(\varphi, \sigma_{1}\right)\left\langle\frac{d N}{d \alpha}(\alpha-\varphi)\right\rangle_{m s} d \varphi \\
& =\int_{-\infty}^{\infty} \rho\left(\varphi, \sigma_{2}\right)\left\langle\frac{d N}{d \alpha}(\alpha-\varphi)\right\rangle d \varphi
\end{aligned}
$$

where $\sigma_{1}$ depends on the distance $\approx\left|x_{e}-x_{c}\right| / \theta\left(x_{e}\right.$ is the exit coordinate) and $\sigma_{2}^{2}=\sigma^{2}+\sigma_{1}^{2}$.

For real beams with the distribution function $\rho_{b}(\varphi)$, we get the following resulting angle distribution:

$$
\rho_{R}(\alpha)=\int_{-\infty}^{\infty} \rho_{b}(\varphi)\left\langle\frac{d N}{d \alpha}(\alpha-\varphi)\right\rangle_{\text {exit }} d \varphi
$$

The limits in the integral from $-\infty$ until $\infty$ have the conditional character [see explanations for Eqs. (21) and (22)]. From the last equations follows

$$
\left\langle\alpha_{R}\right\rangle=\left\langle\alpha_{b}\right\rangle+\left\langle\alpha_{m}\right\rangle+\left\langle\alpha_{v r}\right\rangle,
$$

where $\left\langle\alpha_{R}\right\rangle,\left\langle\alpha_{b}\right\rangle,\left\langle\alpha_{m}\right\rangle$, and $\left\langle\alpha_{v r}\right\rangle$ are the mean values of $\rho_{R}, \rho_{b}, \rho_{m}$, and $\left\langle\frac{d N}{d \alpha}(\alpha)\right\rangle$ distributions, correspondingly. Here the $\left\langle\alpha_{m}\right\rangle$ value requires some explanation. This value is the shift of the mean value of a multiple scattering angle relative to the initial direction (before the single crystal, in other words) of the motion of a charge particle. For amorphous medium, this value is equal to zero due to the fact that the left and right directions are equivalent. In the case of the bent single crystal, the left and right directions are not equivalent and, hence, the $\left\langle\alpha_{m}\right\rangle$ value may differ from zero, in principle. In calculations of the volume reflection effect, we assume that multiple scattering occurs as an amorphous medium. However, we believe if the $\left\langle\alpha_{m}\right\rangle$ value differs from zero essentially, we can see this fact by comparing the calculations and experimental data.

For the dispersions of the corresponding distributions, we get the relation

$$
\sigma_{R}^{2}=\sigma_{b}^{2}+\sigma_{m}^{2}+\sigma_{v r}^{2},
$$

where $\sigma^{2}=\int_{-\infty}^{\infty}(\alpha-\langle\alpha\rangle)^{2} \rho(\alpha) d \alpha$. For the central moment of the third order, we get

$$
M_{R}=M_{b}+M_{m}+M_{v r}
$$

where $M=\int_{-\infty}^{\infty}(\alpha-\langle\alpha\rangle)^{3} \rho(\alpha) d \alpha$. For amorphous me$\operatorname{dia} M_{m}=0$.

\section{GENERALIZATION OF DESCRIPTION}

One can write the equations obtained in this paper in a more general form. It is easy to get from Eq. (15) that

$$
\alpha=\sqrt{2 d / R} f(\xi)
$$

where $f$ is a function of the parameter $\xi=R /\left(E_{0} \beta^{2} d\right)=$ $1 / \delta E$. The multiplier 2 in the equation (under the root sign) is our choice. Thus, the value $2 d / R$ is the angle period $\delta \theta^{2}$ [see Eq. (18)]. Knowing the function $f(\xi)$, one can find the angle of volume reflection for various energies of a particle beam and different bending radii. One can get the $f(\xi)$ function if calculating $\alpha$ for fixed energy $E_{0}$ as a function of the bending radius.

Introducing the critical angle of channeling $\theta_{c}=$ $\sqrt{2 U_{0} /\left(E_{0} \beta^{2}\right)}$, one can write the following relation:

$$
\frac{\alpha}{\theta_{c}}=\frac{1}{\sqrt{\kappa}} f(\kappa)
$$

where $U_{0}$ is the potential barrier and the dimensionless parameter $\kappa$ reads

$$
\begin{aligned}
\kappa & =U_{0} R /\left(\beta^{2} E_{0} d\right)=10 U_{0}[\mathrm{eV}] R[m] /\left(\beta^{2} E_{0}[\mathrm{GeV}] d[\AA]\right) \\
& =U_{0} \xi
\end{aligned}
$$

Here $f$ is the same function as in Eq. (34).

Equations (34) and (35) are valid only for the selected plane of a single crystal [for which the potential function $U(x)$ is given].

\section{CALCULATIONS}

In this section, we demonstrate the applications of obtained relations for calculations of the specific cases of the volume reflection effect. Most of our calculations will be done for the energy of proton beam equal to $400 \mathrm{GeV}$, which was planned in the CERN channeling experiment [13] and for the (110) plane of silicon single crystal. Besides, at the end of this section we will give an illustration of calculations with the use of a generalized parameter.

\section{A. Atomic potential for calculations}

In contrast to the channeling regime, the above-barrier particles move throughout all the points of the potential, and its influence on the motion of the particle arises near critical point $x_{c}$, where the particle is located close to the top of the potential barrier for a long time. Because of this, we think that the correct choice of the one-dimensional atomic potential is an important problem.

Taking into account the aforesaid, we choose the onedimensional atomic potential according to Ref. [19], where potential was calculated on the basis of the measurements of atomic form factors with the use of $x$ rays [20]. For this aim, we use an expansion of the potential in the Fourier 


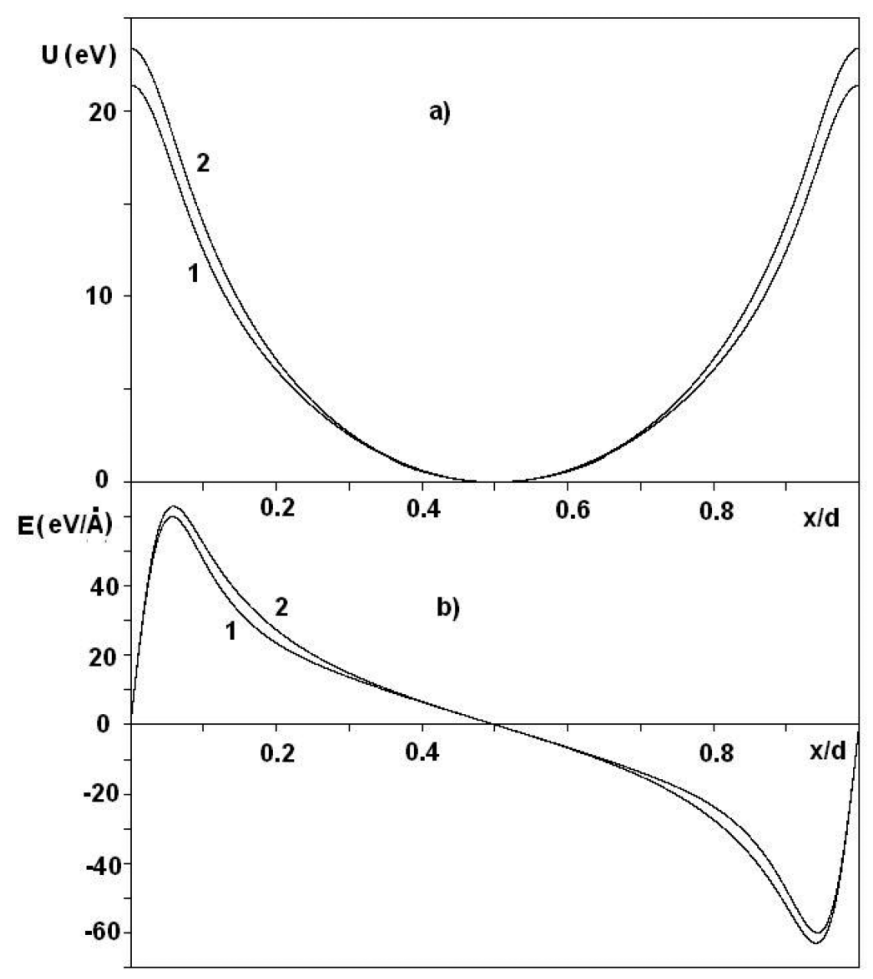

FIG. 2. One-dimensional potential (a) and electric field (b) in the (110) plane of a silicon single crystal (at the room temperature), as functions of the relative coordinate $x / d$, where $d=$ $1.92 \AA$ is the interplanar distance. Curves 1 and 2 correspond to calculations based on the atomic form factors from x-ray measurements and the Moliere model.

series. In a similar manner, we find the one-dimensional electric field and electron density. All these characteristics were calculated at room temperature. It should be noted that this potential was used for comparison of the theoretical description and experimental results for coherent bremsstrahlung of $10 \mathrm{GeV}$ positrons for the (110) plane of silicon single crystal [21], and in this work was shown very good agreement between theory and measurements. Besides, we successfully checked the potential with the use of experimental data [22] which were obtained in the $1.2 \mathrm{GeV}$ electron beam.

We also calculated the one-dimensional potential and electric fields using the Moliere approximation of atomic form factors. The forms of both potentials and electric fields are similar in between, but absolute values for the Moliere potential are larger approximately by $10 \%$ than for potential on the basis of x-ray measurements. So, the potential barriers are equal to $23.36 \mathrm{eV}$ and $21.38 \mathrm{eV}$ for Moliere and above-mentioned approximations, correspondingly (see Fig. 2).

\section{B. Calculation of volume reflection angle}

From general considerations, one can expect that behavior of scattered particles depends on the value of bending radius. Really, at very small radii the effect of volume reflection should disappear due to a dominant value of the linear term in the effective potential. Mathematically, the next condition should be satisfied: $\delta E \gg U_{0}$. From here one can expect that $R_{0}=\beta^{2} E_{0} d / U_{0}$ is some characteristic radius of the process. Our calculations confirm this simple argument.

For $400 \mathrm{GeV}$ proton beam, the characteristic radius is equal to $\approx 3.6 \mathrm{~m}$. The calculations show that at $R>3.6 \mathrm{~m}$ the coherent scattering of particles takes place in one direction (side) relative to their initial motion and at $R<$ $3.6 \mathrm{~m}$ such scattering becomes in both directions. At $R>$ $3.6 \mathrm{~m}$, the incoming particle is deflected in the direction which is opposite to the convexity of bent planes of a single crystal, as shown in Fig. 1. For definiteness, we name this direction the usual one. The angle of the usual direction [calculated according to Eq. (15) and for the coordinate system in Fig. 1] is a negative value, but for convenience of presentation data we define its as the positive one.

Figures 3 and 4 illustrate the calculated volume reflection angle as a function of the transversal energy for several values of the radius. As expected, this function is periodic in accordance with Eq. (16). However, we see that $\alpha(E)$ is a discontinuous function. The purpose is obvious: it is a transition by bound from one potential cell to another. It is significant that function $\alpha(E)$ has a clear maximum and the value of the maximum decreases weakly with the radius decreasing. We have found that the appearance of the maximum (or specifically, when derivative $d \alpha / d E \neq$ 0 ) is connected with the form of the atomic potential. For

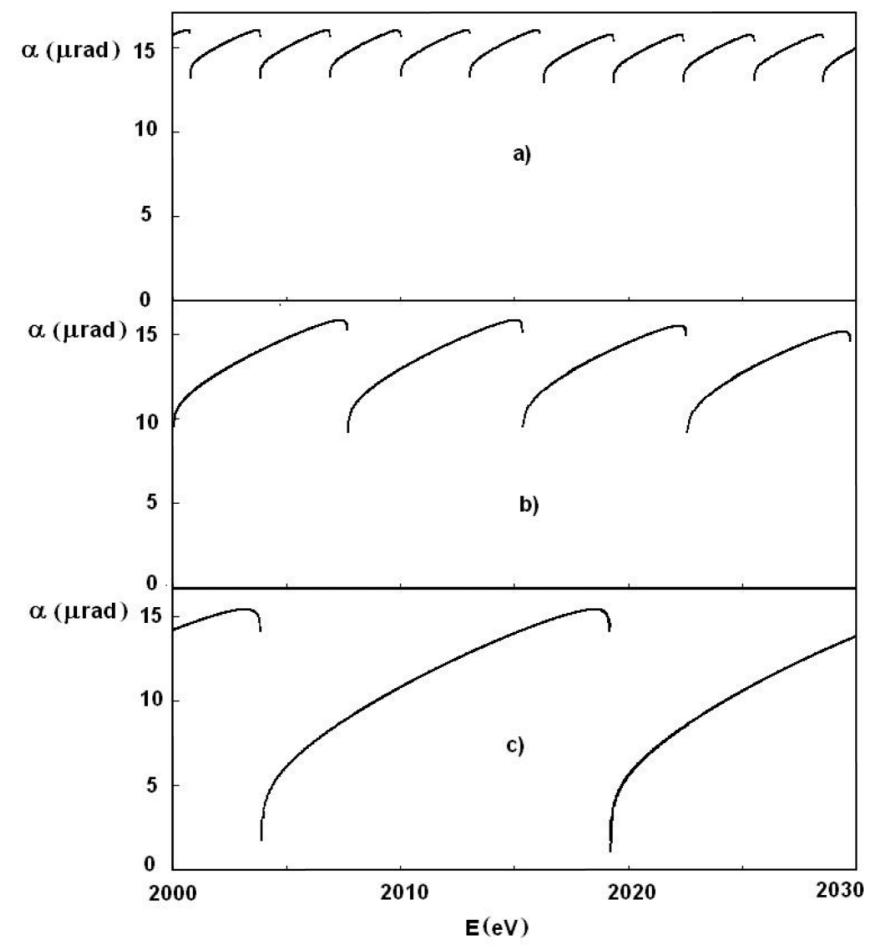

FIG. 3. The angle of volume reflection as a function of the transversal energy for the values of radius equal to $25 \mathrm{~m}$ (a), $10 \mathrm{~m}(\mathrm{~b})$, and $5 \mathrm{~m}(\mathrm{c})$, respectively. 


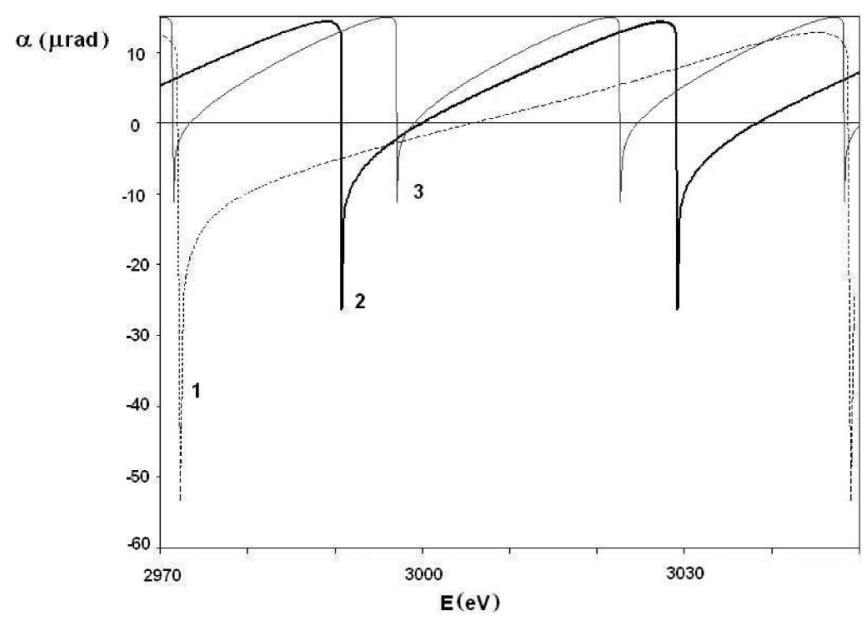

FIG. 4. The angle of volume reflection as a function of the transversal energy for the values of radius equal to $1 \mathrm{~m}, 2 \mathrm{~m}$, and $3 \mathrm{~m}$ (1,2,3 curves, respectively).

some model potentials [such as a parabolic or polynomial [19] (with several numbers of terms) potentials], the maximum coincides with the point of discontinuity. Note that with the decreasing radius the interval of the possible angles increases. It means that the angle divergence is small at large enough radii.

Our calculations (see Fig. 4) show that for small bending radii $(<3.6 \mathrm{~m})$ the scattering takes place in both sides with respect to the initial direction of motion and for negative angles absolute values of these angles in 1.5-3

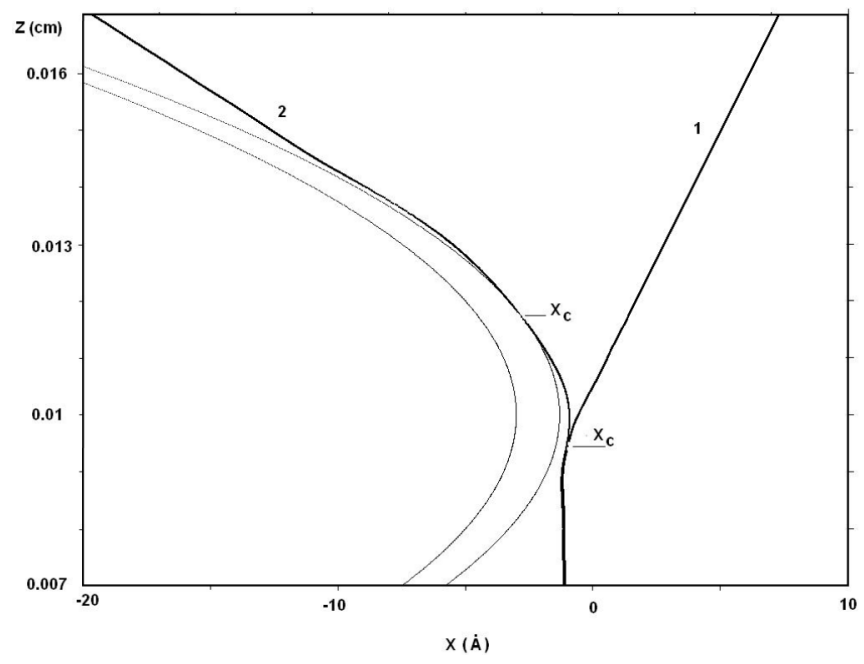

FIG. 5. The picture of volume reflection in a bent single crystal with $R=1 \mathrm{~m}$ and $\theta=100 \mu \mathrm{rad}$. Curves 1 and 2 correspond to maximal absolute values of $\alpha$ (positive and negative, respectively). For visualization, this figure is shown in a turned (on $\theta$ ) coordinate system, or, in other words, the $z$-axis of the new coordinate system is directed along the initial particle velocity. The two ellipses present the location of atoms in a bent single crystal. The elliptic form is due to the difference in scales along $x$ and $z$ axes.

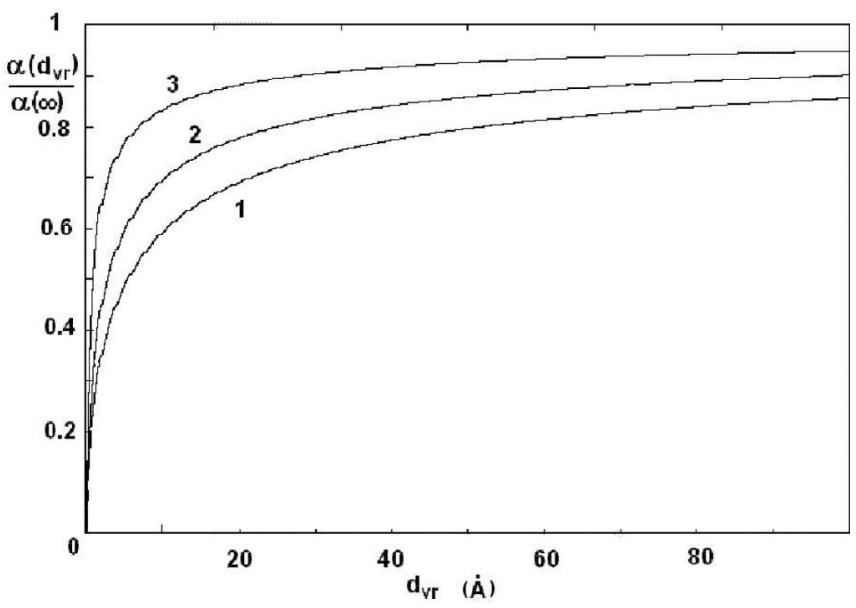

FIG. 6. Calculations of the volume reflection angle according to Eq. (15). The relative value $\alpha\left(d_{v r}\right) / \alpha(\infty)$ as a function of the distance $d_{v r}=\left|x_{0}-x_{c}\right|$. Curves 1,2 , and 3 correspond to $R=$ 25,10 , and $5 \mathrm{~m}$.

times exceed the maximal value of the positive angles (see Fig. 5).

Figure 6 illustrates convergence of the integral in Eq. (15). We see that the main contribution in this integral brings the range $d_{v f} \lesssim 100$ angstroms. One can assume that the process is mainly the result of interaction in the area $\sim \alpha R$ in the longitudinal direction. This estimation corresponds to area $\sim \alpha^{2} R$ in the transversal direction.

\section{Initial distributions of particles}

Knowledge of the function $\alpha(E)$ allows us to calculate the initial distribution of particles $\langle d N / d \alpha(\alpha)\rangle$ over the volume reflection angle. The calculations may be done with the help of Eqs. (19)-(21) or Eq. (26). Obviously, the use of Eq. (26) for calculations is more simple. However, we made a reconstruction of the distribution function by the two methods and we verify that they give the same results. One can find some details of calculations on the basis of Eqs. (19)-(21) in [23].

Figure 7 illustrates the calculated distribution functions for several values of the bending radius. One can see that the distribution functions have a divergence at the maximal angle $\alpha$, where the derivative $d \alpha / d E$ is equal to zero [see Eq. (26)]. At this angle the distribution function $d N / d \alpha$ tends to $+\infty$. However, $\int_{\alpha_{-}}^{\alpha_{+}} d N(\alpha)=1$.

We compared our results of calculation according to Eq. (26) with the calculations by the Monte Carlo method of the same distributions and got a very good agreement in between.

The results of calculations of distribution functions for small bending radii will be presented below.

\section{Entering angle distributions}

As was considered, multiple scattering distorts the initial particle distribution. For calculations of this factor, we can 


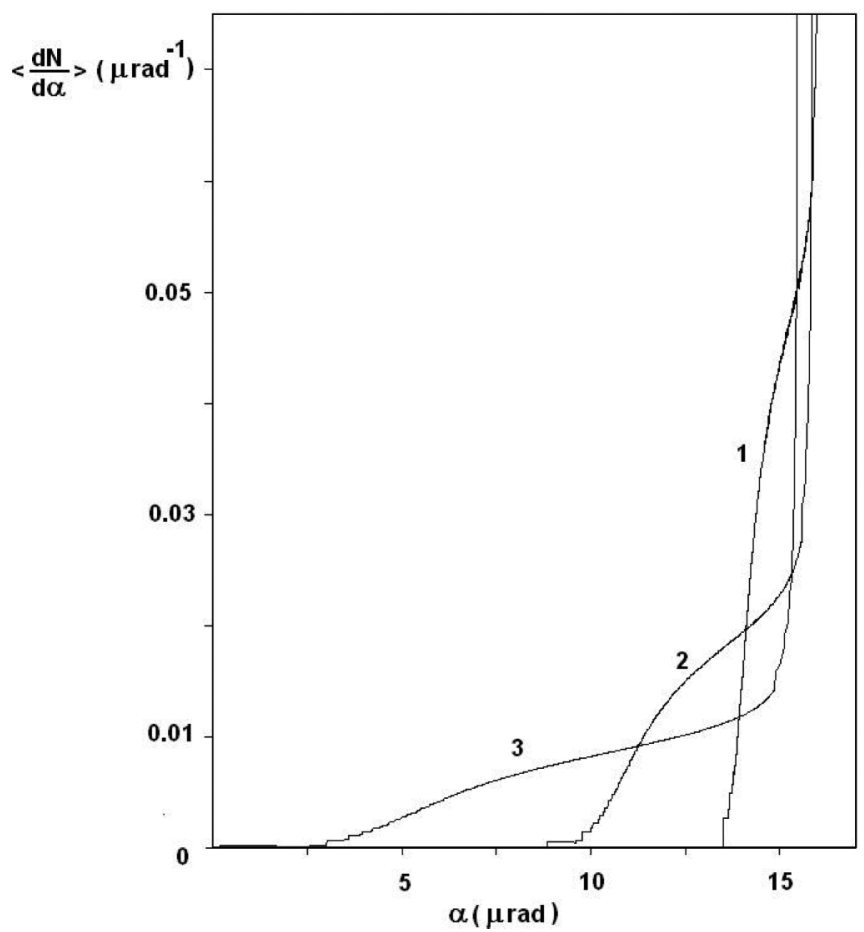

FIG. 7. Distributions of particles over the angle of volume reflection. Curves 1, 2, and 3 correspond to $R=25,10$, and $5 \mathrm{~m}$.

find the distance where the particle is scattered according to relation: $l_{c}=R|\theta|$. Decreasing of angle $|\theta|$ decreases the distance $l_{c}$. On the other hand, the inequality $l_{c} \gg$ $R\left|\theta_{c}\right|$ should be fulfilled. Thus, we can find that the optimal angle $\theta$ lies in the angle range 50-100 $\mu \mathrm{rad}$. It corresponds to $l_{c}=0.125-0.25 \mathrm{~cm}$ for $R=25 \mathrm{~m}$. We also should take into account the distance $l_{d}$ after the critical point (see Fig. 1). Our estimation [see Eqs. (28) and (29)] shows that the distribution form of the entering beam depends on the total distance $l_{s}=l_{c}+l_{d}$ and is practically independent of the relation between $l_{c}$ and $l_{d}$. Figure 8 illustrates the results of calculations of the entering angle distributions at different radii. The calculations are presented for the three thicknesses $l_{s}$ for every radius. They are $0.125,0.25$, and $0.5 \mathrm{~cm}$ for $R=25 \mathrm{~m} ; 0.05,0.1$, and $0.2 \mathrm{~cm}$ for $R=$ $10 \mathrm{~m}$; and $0.025,0.05$, and 0.1 for $R=5 \mathrm{~m}$ (curves 1,2 , and 3 , respectively). We assume the initial (incoming) distribution is physically narrow. From our calculations, one can see that a form of the outgoing angle distributions is close to the Gaussian one.

The angle width of the incident beam in the CERN experiment is expected about some microradians. Then assuming its Gaussian form, it is easy to find the resulting angle distribution after a bent single crystal. At the present time, the precise Monte Carlo calculation of the angle distributions for conditions of the experiment in CERN [13] were made [24]. The first measurement [13] of the mean volume reflection angle for the (110) plane of silicon gave value $[13.9 \pm 0.2$ (stat) \pm 1.5 (syst) $] \mu \mathrm{rad}$. The analytical description and Monte Carlo predict $14.5 \mu \mathrm{rad}$ and

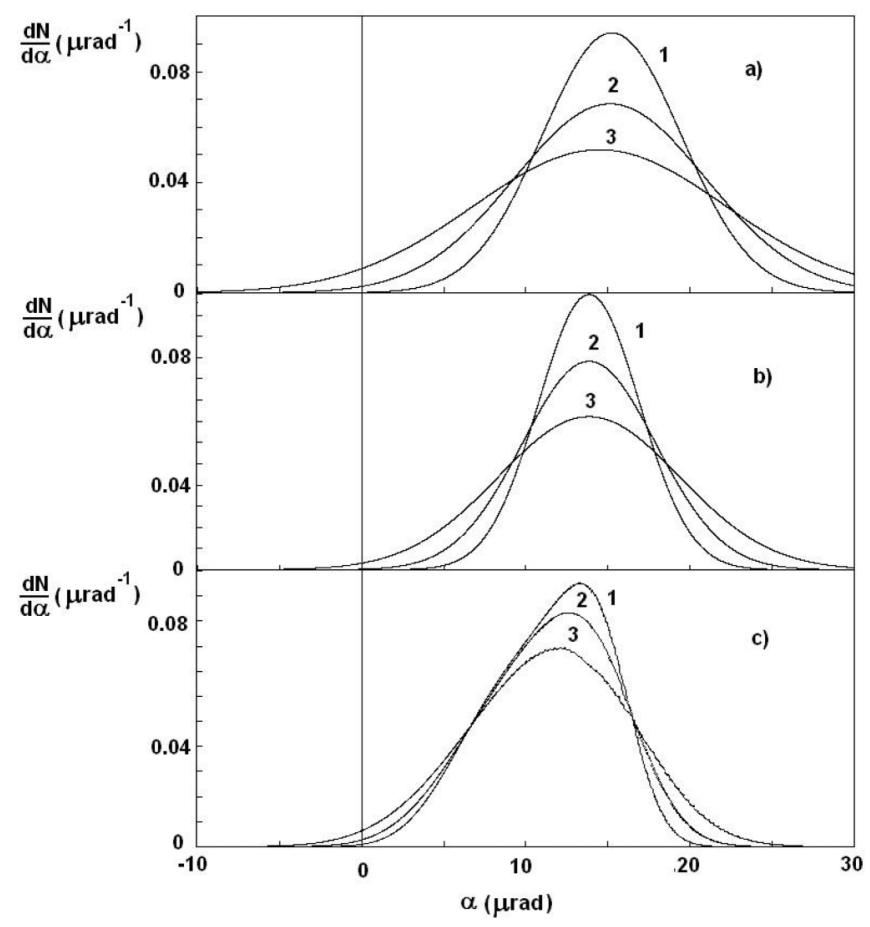

FIG. 8. Distributions of particles after volume reflection and multiple scattering (at the output of a single crystal) as functions of the deflection angle. The zero angle corresponds to the initial direction of a beam. Calculation was made for different radii:

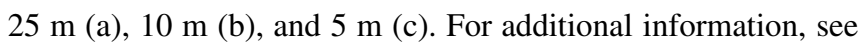
the text.

14.7-15.0 $\mu \mathrm{rad}$, correspondingly [13]. We can say that our analytical description is consistent with experimental result and Monte Carlo simulations.

It should be noted we also investigate the process of volume reflection for negative charged particles. Our analytical description is valid completely for this case (at corresponding well-known changing of a planar potential). In particular, we calculate the angle of volume reflection as a function of the transversal energy. For large enough radii ( $\approx 25 \mathrm{~m}$ ), the result is similar as in Fig. 3 but the value of maximal angle is less in $\approx 1.8$ times. As result at the same conditions, the deflection of negative particles due to volume reflection is more weak in $\approx 1.8$ times.

Besides, we made calculations of the entering distributions for small bending radii $<3.6 \mathrm{~m}$. Figure 9 illustrates these results for $400 \mathrm{GeV}$ positive and negative charged particles. One can see that the mean angle of volume reflection is close to zero $(\approx 1 \mu \mathrm{rad})$. However, the distributions have a large squared mean value of angle (which exceed the similar angle due to multiple scattering in 3-4 times). We see also the long tail in the direction of the negative angles. Thus, the relatively small part of particles may be deflected on a large enough angle in comparison with the case of large bending radii. We can explain this fact by the specific form of the effective potential for small radii (see Ref. [23]). According to our calculations, the part 


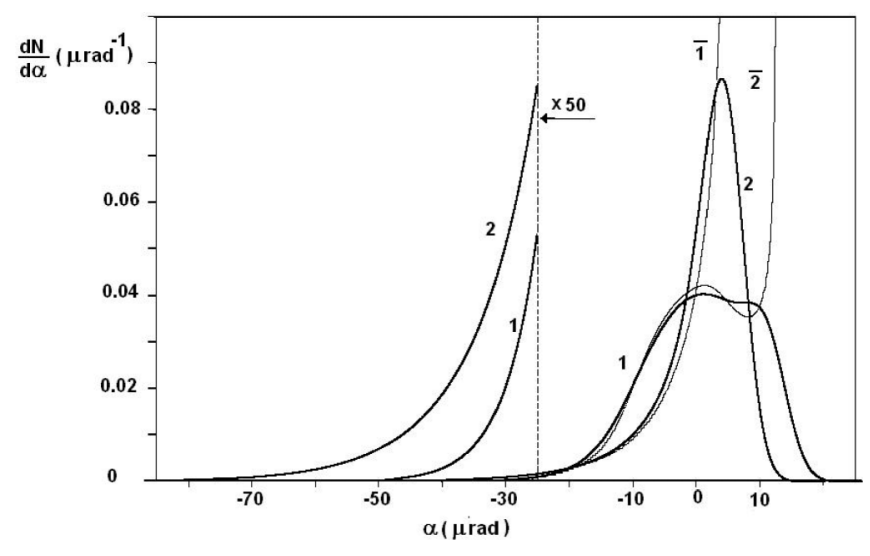

FIG. 9. Distributions of particles after volume reflection and multiple scattering (at the output of a single crystal) as functions of the deflection angle. The zero angle corresponds to the initial direction of a beam. Calculations were made for $R=1 \mathrm{~m}, l_{s}=$ $0.1 \mathrm{~cm}$, and $E_{0}=400 \mathrm{GeV}$. Curves $1(\overline{1})$ and $2(\overline{2})$ correspond to positive and negative particles. Curves $\overline{1}$ and $\overline{2}$ do not take into account multiple scattering. The right tails of distributions are shown in the enlarged scale at 50 times.

of the particles with $|\alpha| \geq 10 \mu \mathrm{rad}$ is $\approx 28 \%$ and $\approx 11 \%$ for positive and negative particles, correspondingly.

It is interesting to note that multiple scattering (in $0.1 \mathrm{~cm}$ of silicon) weakly distorts the volume reflected distribution (except the singular point vicinity).

\section{E. Calculations with the use of generalized parameters}

To this point, we have illustrated the possibilities of our analytical description only for the beam energy equal to $400 \mathrm{GeV}$. Now we will discuss calculations with the use of Eq. (35), which contains the generalized parameter $\kappa$ and is valid for various energies of ultrarelativistic particles. Figures 10 and 11 illustrate the behavior of the mean and squared mean values (divided by the channeling angle $\theta_{c}$ ) of volume reflection angle for positive and negative particles as a function of the parameter $\kappa$. We also present here the following value: $\left(M_{v r}\right)^{1 / 3} / \theta_{c}$, where $M_{v r}$ is the third central moment of distribution function [see Eq. (33)]. This value characterizes a degree of the asymmetry of any distribution. All these values apply only to the pure volume reflection process. Equations (31)-(33) allow one to take into account the angle divergence of a beam and multiple scattering in single crystals.

Calculations were carried out for (110) planes of the silicon and tungsten single crystals at room temperature. For the tungsten single crystal, we use the Moliere model for atomic form factors. One can see that the corresponding characteristics of both crystals are closely related together. Our analysis shows that the visible difference is due to a difference in the form of planar potentials [i.e. due to the difference of $U(x) / U_{0}$-function for various crystals]. Hence, we can conclude that curves presented in Figs. 10 and 11 are approximately universal for a class of simple

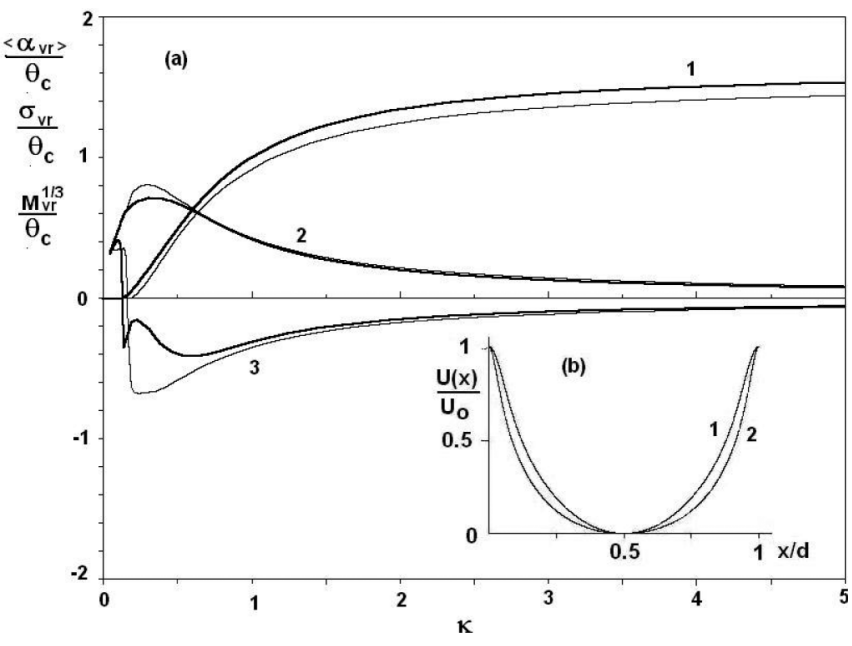

FIG. 10. The main characteristics of volume reflection for positive particles. (a) Values $\left\langle\alpha_{v r}\right\rangle / \theta_{c}, \sigma_{v r} / \theta_{c}$, and $M_{v r}^{1 / 3} / \theta_{c}$ as functions of the generalized parameter $\kappa$ for (110) planes of silicon and tungsten single crystals (the thin and thick curves 1 , 2 , and 3, correspondingly); (b) normalized potentials $U(x) / U_{0}$ of the (110) plane in silicon (1) and tungsten (2) as functions of the relative coordinate.

planar potentials like the (110) plane potential in silicon or tungsten single crystals.

The main peculiarities of the volume reflection process are clearly reflected in the figures and were discussed earlier. So a maximum of the square mean angle takes place at radii slightly more than the critical radius $R_{c}$. It should be noted that the use of value $R / R_{c}$ instead $R / R_{0}$ in parameter $\kappa$ is impaired universality curves for different single crystals.

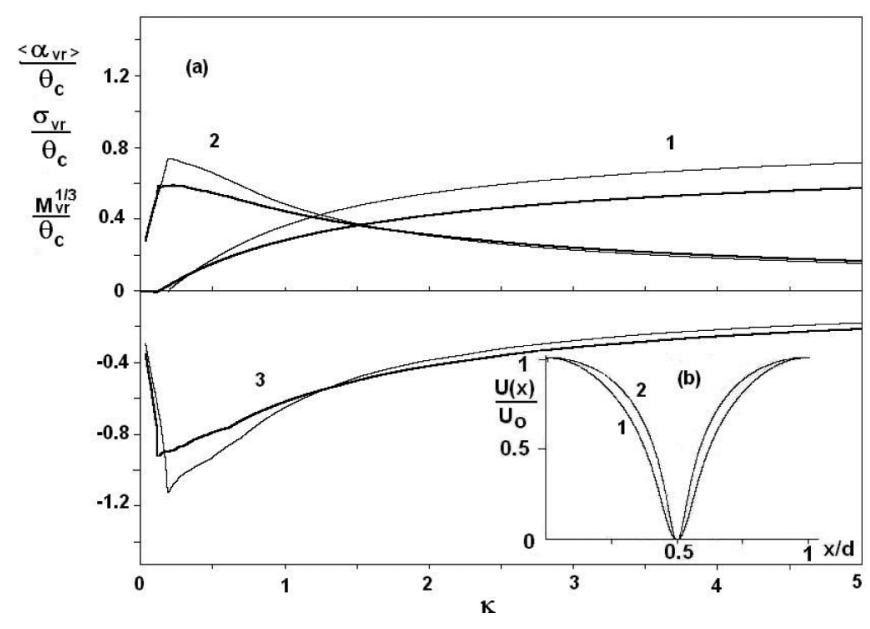

FIG. 11. The main characteristics of volume reflection for negative particles. (a) Values $\left\langle\alpha_{v r}\right\rangle / \theta_{c}, \sigma_{v r} / \theta_{c}$, and $M_{v r}^{1 / 3} / \theta_{c}$ as functions of the generalized parameter $\kappa$ for (110) planes of silicon and tungsten single crystals (the thin and thick curves 1 , 2 , and 3, correspondingly); (b) normalized potentials $U(x) / U_{0}$ of the (110) plane in silicon (1) and tungsten (2) as functions of the relative coordinate. 


\section{CONCLUSIONS}

As considered in the paper, the analytical description of the process of volume reflection of charged particles in bent single crystals allows one to find necessary characteristics of the scattered beam. We think that the proposed method may be useful at different investigations of particle interactions with single crystals. This method may be used in addition to Monte Carlo calculations. Further development of our description is needed: (i) the process of the volume capture of particles in channeling regime should be included in consideration with the aim of the full description of the process; (ii) the extension of the method on single crystals with the variable radius is very desirable.

We suppose that the first point may be realized easy enough because the main cause of the phenomenon is well known. We believe that the second point may be also solved if take into account that, near the critical point, the confined periodicity of the volume reflection angle takes place in some small volume of the bent single crystal.

\section{ACKNOWLEDGMENTS}

The author would like to thank all of the colleagues and participants of the CERN channeling experiment for attention and fruitful discussions. He also thanks V.V. Tikhomirov and A.N. Vasiliev for useful remarks and explanations. This work was partially supported by Grant No. INTAS-CERN 05-103-7525.

\section{APPENDIX: VOLUME REFLECTION IN FINITE SINGLE CRYSTALS}

Here we will consider bent single crystals of a finite thickness. This problem was actual for some samples which were used in CERN experiment [13].

Let us introduce the following definition:

$$
\begin{aligned}
T\left(x, x_{c}\right)= & \int_{x_{0}}^{x_{c}}\left[\frac{1}{\sqrt{\frac{2 c^{2}}{E_{0} \beta^{2}}\left[E-U(x)-E_{0} \beta^{2} x / R\right]}}\right. \\
& \left.-\frac{1}{\sqrt{\frac{2 c^{2}}{E_{0} \beta^{2}}\left[E-U\left(x_{c}\right)-E_{0} \beta^{2} x / R\right]}}\right] d x,
\end{aligned}
$$

where $x_{c}$ is the critical point [see Eq. (9)]. Then in the case of a single crystal with the finite thickness, one can find the angle of volume reflection in the following manner:

$$
\begin{aligned}
\alpha\left(x_{0}, x_{e}\right)= & {\left[v_{x}\left(x_{e}\right)-v_{x}\left(x_{0}\right)+\tilde{v}\left(x_{0}\right)-\tilde{v}\left(x_{e}\right)\right] / c } \\
& +\frac{c}{R}\left[T\left(x_{0}, x_{c}\right)+T\left(x_{e}, x_{c}\right)\right],
\end{aligned}
$$

where $x_{0}$ and $x_{e}$ are the particle coordinates on the entrance in a single crystal and exit from it. If $|\theta|$ is the absolute value of the entrance angle, then

$$
\left|x_{0}-x_{c}\right| \approx R \theta^{2} \text {, }
$$

$$
\left|x_{e}-x_{c}\right| \approx \frac{l^{2}}{R}+R \theta^{2}-2 l|\theta|=R \theta_{1}^{2},
$$

where $l$ is the thickness of a single crystal. For the symmetric case, we have $\left|x_{0}-x_{c}\right|=\left|x_{e}-x_{c}\right|=l^{2} /(4|\theta|)$. If calculated in accordance with Eq. (36), the value $\left|x_{e}-x_{c}\right|$ is negative which means that the volume reflection process is absent.

One can get the following relations:

$$
\begin{aligned}
v_{x}\left(x_{0}\right)-\tilde{v}\left(x_{0}\right) & \approx \sqrt{\frac{2 c^{2}}{E_{0} \beta^{2}}} \frac{\left[U\left(x_{c}\right)-U\left(x_{0}\right)\right]}{2 \sqrt{E-E_{0} \beta x_{0} / R}} \\
& \approx \frac{c \sqrt{R}}{\sqrt{2} E_{0} \beta^{2}} \frac{\left[U\left(x_{c}\right)-U\left(x_{0}\right)\right]}{\sqrt{\left|x_{c}-x_{0}\right|}}, \\
v_{x}\left(x_{e}\right)-\tilde{v}\left(x_{e}\right) & \approx \sqrt{\frac{2 c^{2}}{E_{0} \beta^{2}}} \frac{\left[U\left(x_{c}\right)-U\left(x_{e}\right)\right]}{2 \sqrt{E-E_{0} \beta x_{0} / R}} \\
& \approx \frac{c \sqrt{R}}{\sqrt{2} E_{0} \beta^{2}} \frac{\left[U\left(x_{c}\right)-U\left(x_{e}\right)\right]}{\sqrt{\left|x_{c}-x_{e}\right|}} .
\end{aligned}
$$

These equations are valid at conditions where $E-$ $E_{0} \beta^{2} x_{0} \gg U\left(x_{0}\right)\left[\gg U\left(x_{e}\right)\right]$. It means that the crystal thickness is finite but large enough.

Now we can find the meaning of the first square bracket in Eq. (34):

$$
[\cdots] \approx \frac{\sqrt{R}}{\sqrt{2} E_{0} \beta^{2}}\left[\frac{U\left(x_{c}\right)-U\left(x_{e}\right)}{\sqrt{\left|x_{c}-x_{e}\right|}}-\frac{U\left(x_{c}\right)-U\left(x_{0}\right)}{\sqrt{\left|x_{c}-x_{0}\right|}}\right] .
$$

One can see that for the symmetric case the result is equal to zero. It takes place if the surface nonhomogenities are considerably less than the value $|\theta| d$. However, on the practice this condition is violated. Taking into account this violation, we should consider the values $x_{0}+\xi_{0}$ and $x_{e}+\xi_{e}$ instead of $x_{0}$ and $x_{e}$, where random variables $\xi_{0}$ and $\xi_{e}$ are defined in the range of one period $d$. Under the assumption of the equiprobable distribution of $\xi_{0}$ and $\xi_{e}$ variables, we can find the mean value of Eq. (A3):

$$
\begin{aligned}
\overline{[\cdots]} & \approx \frac{\sqrt{R}}{\sqrt{2} E_{0} \beta^{2}}\left[U\left(x_{c}\right)-\bar{U}\right]\left[\frac{1}{\sqrt{\left|x_{c}-x_{e}\right|}}-\frac{1}{\sqrt{\left|x_{c}-x_{e}\right|}}\right] \\
& =\frac{1}{\sqrt{2} E_{0} \beta^{2}}\left[U\left(x_{c}\right)-\bar{U}\right]\left[1 / \theta-1 / \theta_{1}\right],
\end{aligned}
$$

where $\bar{U}$ is the mean value of the potential.

Our calculations show that the value $U\left(x_{c}\right)$ as a function of the transversal energy $E$ is a practically linear one at large enough quantities of bending radius. Now we can calculate the angle of volume reflection as a function of the transversal energy. The further consideration is analogous to the case of the infinite single crystal.

It should be noted that an additional term [see Eq. (A4)] is equal to zero for the symmetric case, but our study may 
be used for calculations of nonsymmetric passage of charged particles through the finite bent crystals.

[1] A. M. Taratin and S. A. Vorobiev, Phys. Lett. A 119, 425 (1987).

[2] A. M. Taratin and S. A. Vorobiev, Nucl. Instrum. Methods Phys. Res., Sect. B 47, 247 (1990).

[3] R.P. Fliller, A. Drees, D. Gassner, L. Hammos, G. McIntyre, S. Peggs, D. Trbojevic, V. Biryukov, Y. Chesnokov, and V. Terekhov, Phys. Rev. ST Accel. Beams 9, 013501 (2006).

[4] N. V. Mokhov, Channeling Workshop, CERN, 2006.

[5] Yu. M. Ivanov, A. A. Petrunin, V. V. Skorobogatov, Yu. A. Gavrikov, A. V. Gelamkov, L.P. Lapina, A.I. Shchetkovsky, S. A. Vavilov, V.I. Baranov, Yu. A. Chesnokov, A. G. Afonin, V. T. Baranov, V. N. Chepegin, V. Guidi, W. Scandale, and A. Vomiero, Phys. Rev. Lett. 97, 144801 (2006); Yu. M. Ivanov, N. F. Bondar, Yu. A. Gavrikov, A. S. Denisov, A. V. Zhelamkov, V. G. Ivochkin, S. V. Kosianenko, L. P. Lapina, A. A. Petrunin, V. V. Skorobogatov, V. M. Suvorov, A. I. Shchetkovsky, A. M. Taratin, and W. Scandale, JETP Lett. 84, 372 (2006).

[6] W. Scandale, Yu. M. Ivanov, A. Petrunin, V. V. Skorobogatov, Yu. A. Gavrikov, A. V. Zhelamkov. L. P. Lapina, A. I. Shchetkovsky, Yu. A. Chesnokov, V. I. Baranov, V. T. Baranov, V.N. Chepegin, V. Guidi, and A. Vomiero, First Observation of Proton Reflection from Bent Crystals, EPAC, Edinburgh, 2006; Proceedings of EPAC 2006, Edinburg, Scotland, p. 1535.

[7] CERN Channeling Workshop, CERN, 2006, http://indico. cern.ch/conferenceDisplay.py?confId=1183; CERN Channeling Workshop, CERN, 2007, http://indico.cern. $\mathrm{ch} /$ conferenceDisplay.py?confId $=12959$.

[8] W. Scandale, in Proceedings of the LHC Workshop, edited by G. Gartskog and D. Rein (CERN, Switzerland, 1990), Vol. 3, p. 760.

[9] A.D. Kovalenko, A. M. Taratin, and E. N. Tsyganov, Report No. JINR-E1-92-9, 1992.

[10] R. W. Assmann, S. Redaelli, and W. Scandale, Optics Study for a Possible Crystal-based Collimation System for the LHC, EPAC, Edinburgh, 2006; Proceedings of EPAC 2006, Edinburg, Scotland, p. 1526.

[11] I. A. Yazynin, PAC1995, 1996, Vol. 3, pp. 1952-1954.

[12] Yu. A. Chesnokov, Channeling Workshop, CERN, 2006.

[13] W. Scandale, D. A. Still, A. Carnera, G. Della Mea, D. De
Salvador, R. Milan, A. Vomiero, S. Baricordi, P. Dalpiaz, M. Fiorini, V. Guidi, G. Martinelli, A. Mazzolari, E. Milan, G. Ambrosi, P. Azzarello, R. Battiston, B. Bertucci, W. J. Burger, M. Ionica, P. Zuccon, G. Cavoto, R. Santacesaria, P. Valente, E. Vallazza, A. G. Afonin, V. T. Baranov, Yu. A. Chesnokov, V.I. Kotov, V. A. Maisheev, I. A. Yaznin, S. V. Afanasev, A. D. Kovalenko, A. M. Taratin, A.S. Denisov, Yu. A. Gavrikov, Yu. M. Ivanov, V. G. Ivochkin, S. V. Kosyanenko, A. A. Petrunin, V. V. Skorobogatov, V. M. Suvorov, D. Bolognini, L. Foggetta, S. Hasan, and M. Prest, Phys. Rev. Lett. 98, 154801 (2007).

[14] M. Fiorini, P. Dalpiaz, V. Guidi, G. Ambrosi, R. W. Assmann, I. Efthymiopoulos, L. Gatignon, W. Scandale, C. Biino, Yu. A. Chesnokov, Yu. M. Ivanov, R. Santacesaria, A.M. Taratin, and A. Vomiero, Experimental Study of Crystal Channeling at CERNSPS for Beam-halo Cleaning, EPAC, Edinburgh, 2006; Proceedings of EPAC 2006, Edinburg, Scotland, p. 1538.

[15] A. G. Afonin, Channeling Workshop, CERN, 2007.

[16] E. N. Tsyganov, Fermilab Report No. TM-682, TM-684, Batavia, 1976.

[17] X. Artru, S. P. Fomin, N. F. Shul'ga, K. A. Ispirian, and N. K. Zhevago, Phys. Rep. 412, 89 (2005).

[18] V. N. Baier, V.M. Katkov, and V.M. Strakhovenko, Electromagnetic Processes at High Energies in Oriented Single Crystals (World Scientific, Singapore, 1998).

[19] V. A. Maisheev, Nucl. Instrum. Methods Phys. Res., Sect. B 119, 42 (1996).

[20] D. T. Cromer and J. T. Weber, Acta Crystallogr. 18, 104 (1965); 19, 224 (1965).

[21] N. K. Bulgakov, A. S. Vodopianov, J. Wojtkowska, V. M. Golovatyuk, Z. Guzik, N. I. Zimin, I. M. Ivanchenko, R. B. Kadyrov, T. S. Nigmanov, V. D. Ryabtsov, I. A. Tyapkin, N. A. Filatova, E. N. Tsyganov, M.D. Shafranov, A. Forycki, I. N. Ivakhnenko, Yu. V. Nilsen, B. A. Yurev, Richard A. Carrigan, T. Toohig, U. Gibson, Chih-Ree Sun, and R. Wong, JINR Report No. 1-83-621, Dubna, 1983.

[22] I. Endo, M. Harada, K. Kitamara, T. Monaka, Y. Sumi, M. Tobiyama, H. Tsujikawa, K. Watanabe, K. Yoshida, T. Ohba, K. Baba, and H. Motegi, Phys. Rev. Lett. 60, 2292 (1988).

[23] V. A. Maisheev, arXiv:physics/0607009. This preprint is the first version of the present paper. The final version presented here is widely different. One can say that both versions complement each other.

[24] A. M. Taratin, Channeling Workshop, CERN, 2006. 